\title{
A!
}

This is an electronic reprint of the original article.

This reprint may differ from the original in pagination and typographic detail.

Trukhin, V.N.; Bouravleuv, A.D.; Mustafin, I.A.; Kakko, Joona-Pekko; Huhtio, Teppo; Cirlin, G.E.; Lipsanen, Harri

\section{Generation of terahertz radiation in ordered arrays of GaAs nanowires}

Published in:

Applied Physics Letters

DOI:

$10.1063 / 1.4923211$

Published: 01/01/2015

Document Version

Publisher's PDF, also known as Version of record

Please cite the original version:

Trukhin, V. N., Bouravleuv, A. D., Mustafin, I. A., Kakko, J-P., Huhtio, T., Cirlin, G. E., \& Lipsanen, H. (2015). Generation of terahertz radiation in ordered arrays of GaAs nanowires. Applied Physics Letters, 106(25), 1-4. [252104]. https://doi.org/10.1063/1.4923211

This material is protected by copyright and other intellectual property rights, and duplication or sale of all or part of any of the repository collections is not permitted, except that material may be duplicated by you for your research use or educational purposes in electronic or print form. You must obtain permission for any other use. Electronic or print copies may not be offered, whether for sale or otherwise to anyone who is not an authorised user. 


\section{Generation of terahertz radiation in ordered arrays of GaAs nanowires}

V. N. Trukhin, A. D. Bouravleuv, I. A. Mustafin, J. P. Kakko, T. Huhtio, G. E. Cirlin, and H. Lipsanen

Citation: Appl. Phys. Lett. 106, 252104 (2015); doi: 10.1063/1.4923211

View online: http://dx.doi.org/10.1063/1.4923211

View Table of Contents: http://aip.scitation.org/toc/apl/106/25

Published by the American Institute of Physics

\section{Articles you may be interested in}

Terahertz generation by GaAs nanowires

Applied Physics Letters 103, 072108 (2013); 10.1063/1.4818719

Growth of Au-catalyzed ordered GaAs nanowire arrays by molecular-beam epitaxy

Applied Physics Letters 81, 5177 (2002); 10.1063/1.1532772

Protective capping and surface passivation of III-V nanowires by atomic layer deposition

AIP Advances 6, 015016 (2016); 10.1063/1.4941063

Perspective: Ultrafast magnetism and $\mathrm{THz}$ spintronics

Journal of Applied Physics 120, 140901 (2016); 10.1063/1.4958846

Terahertz emission from GaAs-AIGaAs core-shell nanowires on Si (100) substrate: Effects of applied magnetic field and excitation wavelength

Applied Physics Letters 102, 063101 (2013); 10.1063/1.4791570

Catalyst-free growth of GaAs nanowires by selective-area metalorganic vapor-phase epitaxy

Applied Physics Letters 86, 213102 (2005); 10.1063/1.1935038

\section{A IP $\mid$ Applied Physics} Letters

Save your money for your research.

It's now FREE to publish with us no page, color or publication charges apply.

If your article has the

potential to shape the future of applied physics, it BELONGS in Applied Physics Letters 


\title{
Generation of terahertz radiation in ordered arrays of GaAs nanowires
}

\author{
V. N. Trukhin, ${ }^{1,2}$ A. D. Bouravleuv, ${ }^{1,3,4}$ I. A. Mustafin, ${ }^{1,2}$ J. P. Kakko, ${ }^{5}$ T. Huhtio, ${ }^{5}$ \\ G. E. Cirlin, ${ }^{1,3,4}$ and H. Lipsanen ${ }^{5}$ \\ ${ }^{1}$ Ioffe Institute, 194021 St. Petersburg, Russia \\ ${ }^{2}$ ITMO University, 197101 St. Petersburg, Russia \\ ${ }^{3}$ St. Petersburg Academic University, 194021 St. Petersburg, Russia \\ ${ }^{4}$ Department of Physics, St. Petersburg State University, 199034 St. Petersburg, Russia \\ ${ }^{5}$ Department of Micro and Nanosciences, Aalto University, FIN-00076 Aalto, Finland
}

(Received 25 March 2015; accepted 17 June 2015; published online 25 June 2015)

\begin{abstract}
$\mathrm{THz}$ generation under excitation by ultrashort optical pulses in ordered arrays of GaAs nanowires is reported. It was found that the efficiency of $\mathrm{THz}$ radiation generation increases due to the resonant leaky mode excitation in nanowires. The maximum value of the $\mathrm{THz}$ field is achieved when the distance between the nanowires is of the order of the wavelength of exciting light. (C) 2015 AIP Publishing LLC.

[http://dx.doi.org/10.1063/1.4923211]
\end{abstract}

Semiconductor nanostructures, such as freestanding semiconductor nanowires (NWs), are one of the most promising low-dimensional nano-objects for applications in nanoelectronics, nanophotonics, and nanobioelectronics. In addition, the use of such quasi-1D nanostructures, due to their unique electrical and optical properties, is an interesting direction to improve existing $\mathrm{THz}$ emitters. Recent studies have shown that the efficiency of a $\mathrm{THz}$ emitter can be substantially improved if the surface-to-volume ratio of the structure is increased. ${ }^{1-7}$ However, the real increase in the efficiency of $\mathrm{THz}$ generation in such structures with structured surface, compared to the $\mathrm{THz}$ generation in InAs bulk, has not been demonstrated. It is well known that the $\mathrm{THz}$ generation in a semiconductor under excitation by ultrashort optical pulses can be caused by two processes: short-term movement of photocarriers propagating near the surface $^{8,9}$ and nonlinear optical processes. ${ }^{10}$ In this paper, we present the experimental results of $\mathrm{THz}$ generation in ordered arrays of GaAs NWs with different fill factors under excitation by ultrashort optical pulses.

The GaAs NW arrays were grown by selective-area epitaxy (SAE) using a horizontal Thomas Swan metal-organic vapour phase epitaxy (MOVPE) system on p-type GaAs (111)B substrates. An Oxford Systems Plasmalab 80plus plasma enhanced chemical vapour deposition (PECVD) was used on clean substrates to deposit a $40 \mathrm{~nm}$ thick $\mathrm{SiO}_{\mathrm{x}}$ layer that acts as the growth mask for SAE. A Vistec EPBG5000pES electron beam lithography (EBL) system and reactive-ion etching (RIE, Oxford Systems Plasmalab 80Plus) were used to pattern openings $\left(d_{0}\right)$ of $50 \mathrm{~nm}$ and $100 \mathrm{~nm}$ to the $\mathrm{SiO}_{\mathrm{x}}$ layer. Several $200 \mu \mathrm{m} \times 200 \mu \mathrm{m}$ arrays of holes with both opening sizes were patterned into a triangular lattice with varying pitch (a) from $300 \mathrm{~nm}$ to $2100 \mathrm{~nm}$ with a $300 \mathrm{~nm}$ step size. Prior to transferring the samples into the MOVPE reactor, the regular degreasing and cleaning steps were performed in acetone, isopropanol, and de-ionized water. Before the NW growth, the samples were thermally cleaned at $750{ }^{\circ} \mathrm{C}$ under $\mathrm{H}_{2}$ flow in the reactor for $5 \mathrm{~min}$. The growth was performed using trimethylgallium (TMGa) and tertiarybutylarsine (TBAs) precursors with $\mathrm{H}_{2}$ carrier gas (total gas flow $5 \mathrm{slm}$ ) at $750^{\circ} \mathrm{C}$ for $5 \mathrm{~min}$. The TMGa and TBAs flows were $0.83 \mu \mathrm{mol} / \mathrm{min}$ and $210.1 \mu \mathrm{mol} / \mathrm{min} \quad(\mathrm{V} / \mathrm{III}=253)$, respectively.

Fig. 1 shows $20^{\circ}$ tilted scanning electron microscope (SEM) images of the fabricated GaAs NW arrays. The NWs are positioned where the openings were patterned. They have vertical sidewalls and exhibit a hexagonal morphology. As shown in Fig. 1, the heights and diameters of the NWs depend on the opening size and array pitch $(a)$. The $50 \mathrm{~nm}$ and $100 \mathrm{~nm}$ openings resulted in approximately $80 \mathrm{~nm}$ and $160 \mathrm{~nm} \mathrm{NW}$ diameter $(d)$, respectively, while the heights increased with increasing pitch. Average heights (h) for the NW arrays with $100 \mathrm{~nm}$ opening are listed in the inset of Fig. 3.

The experiments were performed using time-domain spectroscopy which is able to detect the electric field amplitude and phase of THz radiation. A Ti:sapphire femtosecond laser was used as the source for photoexcitation of nanostructures. It generated $15 \mathrm{fs}$ light pulses at a repetition rate of $80 \mathrm{MHz}$. The central wavelength of the light pulse was equal to $795 \mathrm{~nm}$. The sample was illuminated at an oblique incidence. The emitted $\mathrm{THz}$ radiation was collected by reflection geometry (see Fig. 2(a)). The detailed information concerning experimental setup is presented in the article. ${ }^{11}$

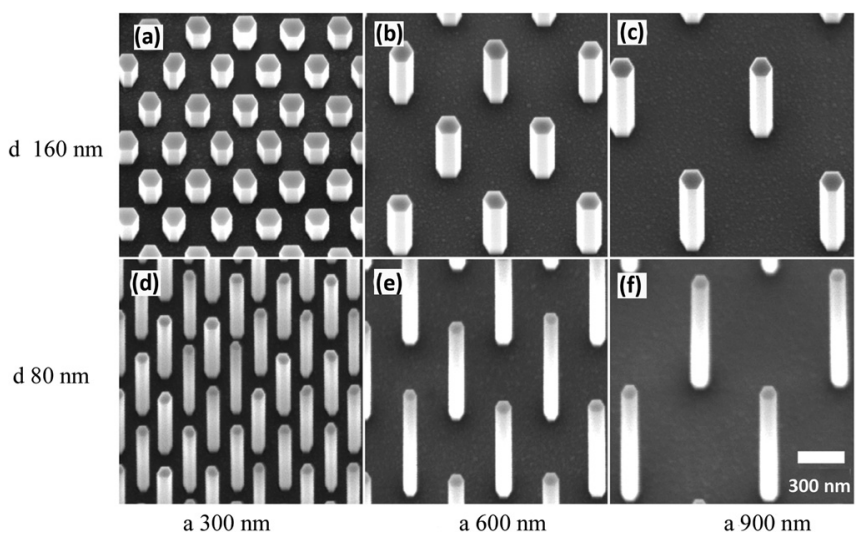

FIG. $1.20^{\circ}$ tilted SEM images of the GaAs NW arrays. The top row ((a)-(c)) has opening size of $100 \mathrm{~nm}$, while the bottom row ((d)-(f)) have opening size of $50 \mathrm{~nm}$. The columns have array pitch of $300 \mathrm{~nm}, 600 \mathrm{~nm}$, and $900 \mathrm{~nm}$, respectively. The scale bar in (f) applies to all images. 
Incident light

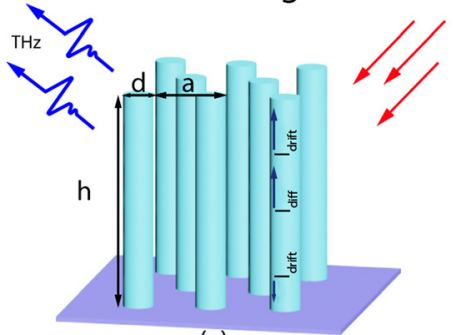

(a)

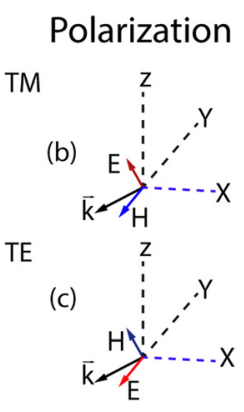

FIG. 2. (a) The scheme of structure with ordered GaAs NWs under oblique incident illumination, which illustrates the various current mechanisms; (b) the illumination geometry for transverse-magnetic polarization; (c) the illumination geometry for transverse-electric polarization.

Fig. 3 shows the waveforms of the THz pulses obtained from NW arrays with a diameter of approximately $160 \mathrm{~nm}$ and different densities. Spectrum of $\mathrm{THz}$ pulses generated by an array of NWs with $1200 \mathrm{~nm}$ pitch is shown in the left inset. The average height and pitch of the arrays are shown in the right inset. It should be noted that NW arrays with different densities were grown simultaneously on the same substrate. Nevertheless, the NW heights were found to be different.

The maximum amplitude of the $\mathrm{THz}$ pulse electric field was observed to correspond to the NW array with $a=1200 \mathrm{~nm}$ (see Fig. 3), whereas the generation efficiency decreased as the distance between the NWs differed from this value. Besides, it was found that there was a sign inversion of the $\mathrm{THz}$ pulse field amplitude for NWs with heights equal to $557 \mathrm{~nm}$. It should be noted that the sign of the $\mathrm{THz}$ field generated from the bulk p-type GaAs substrate had the same polarity, but the amplitude of the field was much smaller.

As was reported earlier, ${ }^{7}$ the generation of $\mathrm{THz}$ radiation in GaAs NWs excited by ultrashort optical pulses with the photon energies greater than the band gap of the semiconductor can be caused by the current of photoexcited carriers, which is associated with their movement in the surface electric field or in the internal field, as well as ambipolar

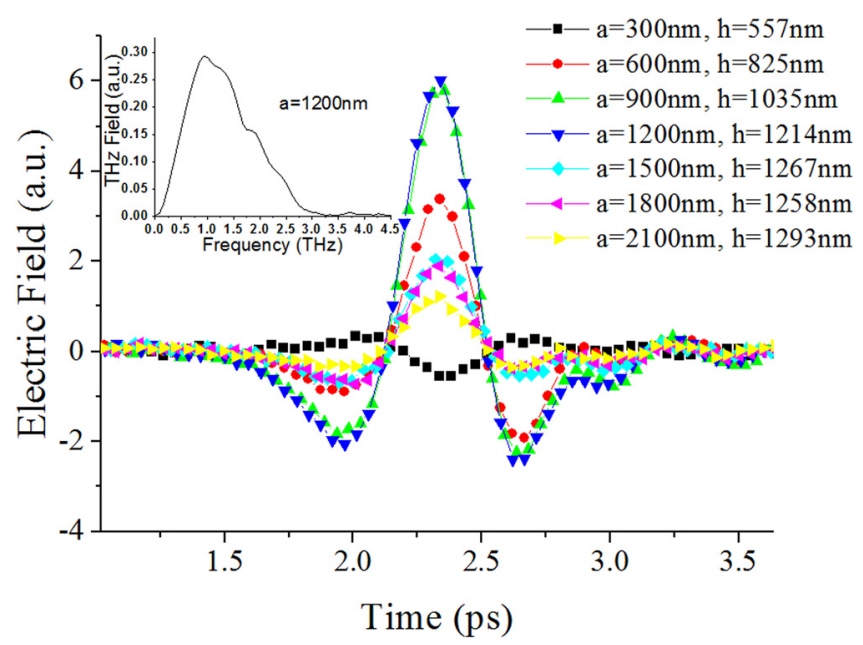

FIG. 3. Waveforms of the THz pulses for different NW arrays $(d=160 \mathrm{~nm})$. Spectrum of THz pulses generated by an array of NWs with $1200 \mathrm{~nm}$ pitch is shown in the left inset. diffusion of charge carriers under inhomogeneous excitation. It seems that the surface field in our samples should be negligible, since the electrons can be fully captured by the surface states. The surface potential of a NW with a diameter of about $160 \mathrm{~nm}$, and taking into account that the background carrier concentration equals to $10^{16} \mathrm{~cm}^{-3}$, will be of the order of thermal energy, kT. ${ }^{12}$ Thus, the generation of $\mathrm{THz}$ radiation appears to be determined by a process that is not correlated to the movement of charge carriers in the surface field. The emergence of anisotropy in the momentum distribution of charge carriers due to their reflection from the upper face of the NW could be such a process. This anisotropy leads to a ballistic current of electrons moving to the bottom of the NW. As a consequence, there is a "reactive electromotive force"13 that can result in non-equilibrium plasma oscillations of the charge carriers.

It should be noted that since the NWs have n-type conductivity, whereas the substrates are p-type, a contact field exists between the NWs and the substrate. Thus, the generation of $\mathrm{THz}$ radiation in our samples can be associated with the occurrence of anisotropy in the momentum distribution of charge carriers, as well as with the movement of charge carriers in the contact field and ambipolar diffusion. It should be emphasized that the contribution of the ambipolar diffusion appears to be negligible if the excitation wavelength is greater than the distance between the nanowires.

The direction of motion of the charge carriers in the $p-n$ junction field is opposite to the direction of the carriers associated with the occurrence of anisotropy in the momentum distribution of charge carriers (see Fig. 2). Therefore, $\mathrm{THz}$ fields generated by these currents will also have opposite directions of the electric field. As a result, the total $\mathrm{THz}$ field that is registered in the wave zone will decrease with an increase of the drift current contribution in the $\mathrm{p}-\mathrm{n}$ junction.

The observed effect of the THz field sign inversion with decreasing NW height (h) shows that for $h>600 \mathrm{~nm}$ the ballistic motion contribution of non-equilibrium charge carriers can be larger; and therefore the direction of the $\mathrm{THz}$ field is opposite to the direction of the $\mathrm{THz}$ field generated by the bulk p-type GaAs substrate. At smaller NW heights, the drift motion contribution of non-equilibrium charge carriers in the $\mathrm{p}-\mathrm{n}$ junction increases and, as a consequence, the direction of the THz field changes. It is obvious that with increasing excitation power separable carriers will screen the internal fields. It leads to unequal contribution to the $\mathrm{THz}$ photocurrent generation in these fields. It can greatly affect the dependence of the peak amplitude of $\mathrm{THz}$ pulse on the excitation power. Fig. 4 shows the above-mentioned dependence for an array of NWs with the following parameters: $a=900 \mathrm{~nm}$, $d=160 \mathrm{~nm}$, and $h=1035 \mathrm{~nm}$. The polarization direction of the exciting light was in a plane parallel to both the NW axis and the wave vector of the incident wave. The observed dependence has nonlinear behavior; it reaches saturation with increasing excitation power.

To determine the mechanism of $\mathrm{THz}$ generation from NW arrays, first of all, it is necessary to investigate the dependence of the $\mathrm{THz}$ field peak amplitude on the array fill factor with equally long NWs. This dependence was obtained for an array of NWs with diameters $d=80 \mathrm{~nm}$ and heights $h \sim 1100 \mathrm{~nm}$ (see Fig. 5). The obtained data 


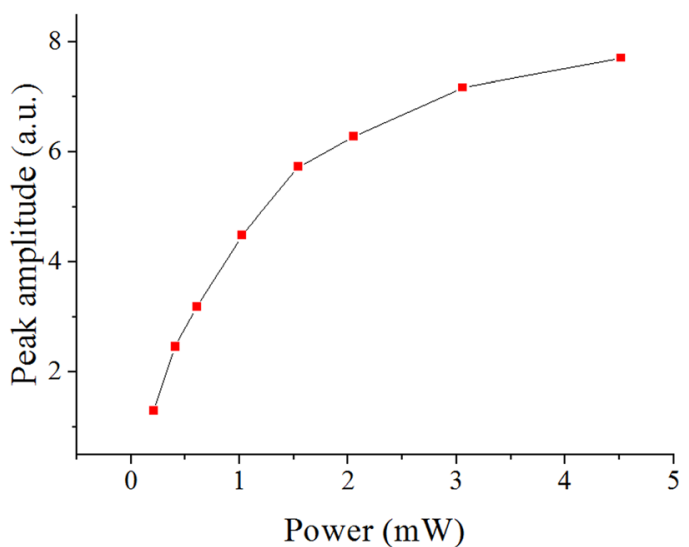

FIG. 4. Dependence of the maximum amplitude of the THz pulse waveform on the excitation power. The area of the light spot is about $6400 \mu \mathrm{m}^{2}$.

demonstrated that the maximum efficiency of the $\mathrm{THz}$ radiation generation was observed when the NW pitch $a$ was in the order of the wavelength of the exciting light $(\lambda=795 \mathrm{~nm})$. At $a>\lambda$ (low fill factor), as shown in Fig. 5, there is a linear dependence. It indicates that in this case the efficiency of $\mathrm{THz}$ generation was proportional to the NW density. Thus, the $\mathrm{THz}$ field registered in the wave zone is the sum of the contributions of electromagnetic radiation from each NW. It should be noted that a single NW can be represented as a generating point dipole, since both $d$ and $h \ll \lambda_{\mathrm{THz}}$.

However, if $a<\lambda$ (high fill factor), THz field generated by an array of NWs decreases. Therefore, the assumption expressed by the authors of several publications ${ }^{4-6}$ about the significant growth of $\mathrm{THz}$ generation efficiency with an increase of the fill factor when $a<\lambda$ seems to be untenable. In this case, the behavior of the $\mathrm{THz}$ field peak amplitude on the NW array filling factor (see Fig. 5) seems to be explained by the results obtained from the investigation of the dependence of the $\mathrm{THz}$ generation on the exciting radiation polarization. It was found that at certain parameters of NWs and the exciting light the $\mathrm{THz}$ generation efficiency becomes larger with excitation polarization perpendicular to the NW axis.

Fig. 6 shows the ratio between the maximum amplitude of the $\mathrm{THz}$ pulse for transverse-magnetic (TM)- and transverse-electric (TE)-polarization of the exciting radiation

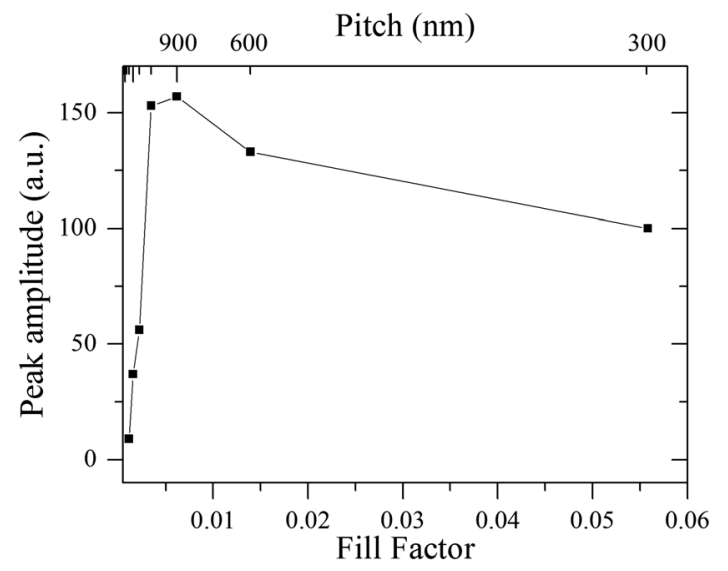

FIG. 5. Dependence of the maximum amplitude of the THz field on the NW array fill factor.

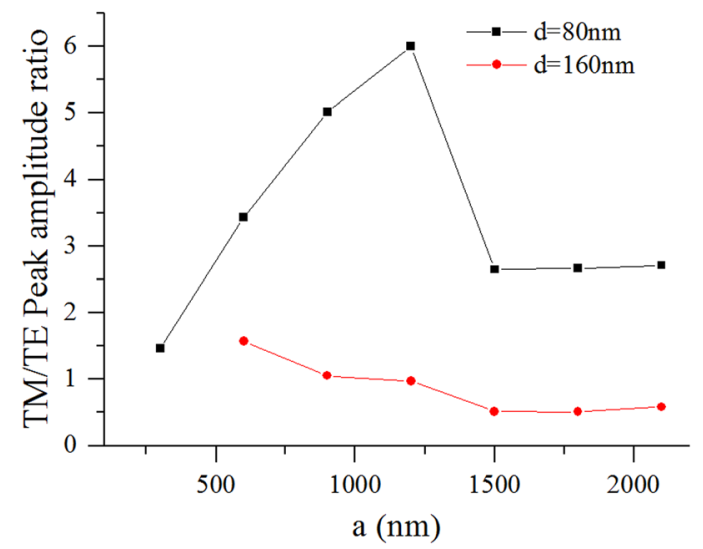

FIG. 6. Dependence of the ratio between the maximum amplitude of $\mathrm{THz}$ pulse for the TM polarization and TE polarization for NWs with diameters equal to $160 \mathrm{~nm}$ (squares) and $80 \mathrm{~nm}$ (circles) on the NW array pitch.

as a function of the NW array pitch. The angle of exciting light incidence was equal to $45^{\circ}$. According to the data obtained, if $a \gg \lambda$, the maximum THz field amplitude from an array of NWs with diameters $d=160 \mathrm{~nm}$ is approximately two times larger for the TE polarization than for the TM polarization. In contrast, for an array of NWs with diameter $d=80 \mathrm{~nm}$, the peak amplitude of the TM polarization is two times larger than that of the TE polarization. These experimental results are well-described in terms of the mechanism associated with the leaky mode excitation at oblique incidence of light onto dielectric cylinder. In the articles ${ }^{14,15}$ based on the theory of Lorenz-Mie, it was shown that when an electromagnetic plane wave falls onto an infinitely long dielectric cylinder, the resonant excitation of leaky modes occurs. The leaky modes are the cylindrical waveguide modes whose fields extend outside of the structures. ${ }^{16}$ In the case of an absorbing medium, the light absorption will occur in resonance as well. Therefore, the field amplitude inside the cylinder can exceed the value of the incident field by several orders of magnitude under certain conditions. It should be noted that such resonant absorption was observed in the $\operatorname{article}^{17}$ where the photovoltaic properties of structures based on GaAs NWs was investigated. Using data for the complex refractive index of GaAs, we can calculate the cross-section for the light absorption at oblique incidence on the cylinder. ${ }^{18}$

Fig. 7 demonstrates the calculated dependence of the absorption efficiency of light at an incidence angle of $45^{\circ}$ on the characteristic value $k r$, where $k$ is the wave vector of the excited light and $r$ is the NW radius. The light absorption was approximately two times greater for the TE-polarization as compared to the TM- polarization at a value of $k r=0.63$ $(d=160 \mathrm{~nm})$. The opposite behaviour was observed when $k r=0.31(d=80 \mathrm{~nm})$. The magnitude of the THz field generated by the movement of non-equilibrium charge carriers in the near-surface or in the applied electric field is proportional to the concentration of photoexcited charge carriers. ${ }^{19}$ Accordingly, the magnitude of the $\mathrm{THz}$ field will correlate with the amount of the absorbed light. Thus, the experimental results shown in Fig. 6 can indeed be described in terms of the leaky mode excitation in NWs. Moreover, in the framework of this mechanism, the experimental results 


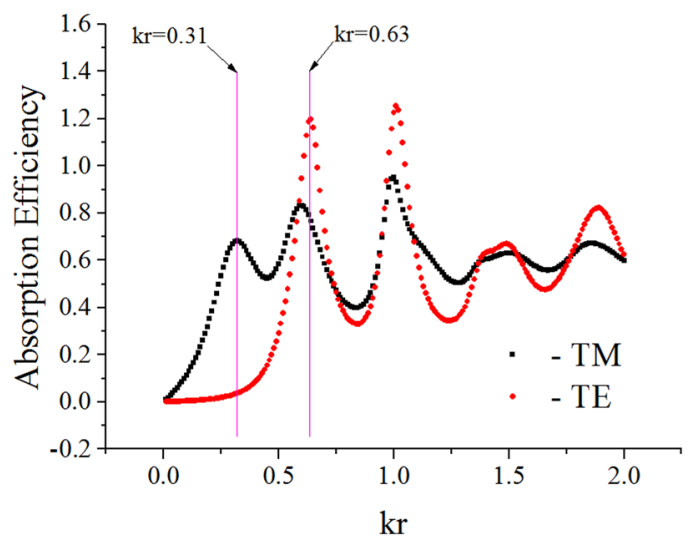

FIG. 7. Spectra of the absorption efficiency for TM and TE polarizations in an incident angle of $45^{\circ}$.

associated with the determination of the maximum efficiency of $\mathrm{THz}$ generation by the NW array can be explained.

It was shown in Ref. 20 that the frequency of leaky modes in the NWs and their quality factor begins to change when the distance between NWs becomes smaller than the wavelength. This change was associated with the near-field interaction between NWs. The absorption efficiency in the NWs also seems to vary. The experimental results demonstrated in Fig. 5 are also confirmed the near-field interaction of the excited modes. Besides, the layer with NWs can be considered as a photonic crystal if the density of NWs is high and they have periodic arrangement. In this case, the photonic modes can be excited. ${ }^{21}$

Therefore, the maximum efficiency of the THz generation by an array of NWs seems to be at the condition of $\lambda \sim a$ and with an appearance of the resonant excitation of the cylindrical waveguide modes.

Thus, the investigation of the $\mathrm{THz}$ radiation generation by arrays of GaAs NWs with different geometrical parameters has been performed under excitation by ultrashort optical pulses. It is shown that the efficiency of $\mathrm{THz}$ generation increases due to the resonant excitation of leaky modes in a NW. The maximum THz field amplitude is achieved when the distance between the NWs is in the order of the exciting light wavelength, as well as the corresponding values of the
NW diameter. Therefore, the use of ordered NW arrays can lead to the creation of highly effective $\mathrm{THz}$ sources.

This work has been supported in part by the RFBR (Grant No. 14-02-00957-a), by the Program of the Presidium of RAS, and by Aalto Energy Efficiency Program. A.D.B. and G.E.C. acknowledge the support from the Skolkovo Foundation (Grant Agreement No. 4 dd. 25.12.2014).

${ }^{1}$ M. Reid, I. V. Cravetchi, R. Fedosejevs, I. M. Tiginyanu, and L. Sirbu, Appl. Phys. Lett. 86, 021904 (2005).

${ }^{2}$ H. Ahn, Y.-P. Ku, Y.-C. Wang, C.-H. Chuang, S. Gwo, and Ci-Ling Pan, Appl. Phys. Lett. 91, 132108 (2007).

${ }^{3}$ S. He, X. Chen, X. Wu, W. Gang, and F. Zhao, J. Lightwave Technol. 26, 1519 (2008).

${ }^{4}$ J. G. Bok, C. Y. Jae, M. Yoon, K. H. Sung, S. Y. Suk, P. Jeunghee, and K. Chul, Opt. Express 18, 16353 (2010).

${ }^{5}$ P. Hoyer, M. Theuer, R. Beigang, and E.-B. Kley, Appl. Phys. Lett. 93, 091106 (2008).

${ }^{6}$ D. V. Seletskiy, M. P. Hasselbeck, J. G. Cederberg, A. Katzenmeyer, M. E. Toimil-Molares, F. Léonard, A. Alec Talin, and M. Sheik-Bahae, Phys. Rev. B 84, 115421 (2011).

${ }^{7}$ V. N. Trukhin, A. S. Buyskih, L. L. Samoilov, Yu. B. Samsonenko, G. E. Cirlin, M. A. Kaliteevski, and A. J. Gallant, Appl. Phys. Lett.103, 072108 (2013).

${ }^{8}$ X.-C. Zhang, J. T. Darrow, B. B. Hu, D. H. Auston, M. T. Schmidt, P. Tham, and E. S. Yang, Appl. Phys. Lett. 56, 2228 (1990).

${ }^{9}$ J. E. Pedersen, I. Balslev, J. M. Hvam, and S. R. Keiding, Appl. Phys. Lett. 61, 1372 (1992).

${ }^{10}$ S. L. Chuang, S. Schmitt-Rink, B. I. Greene, P. N. Saeta, and A. F. J. Levi, Phys. Rev. Lett. 68, 102 (1992).

${ }^{11}$ V. N. Trukhin, A. V. Andrianov, V. A. Bykov, A. O. Golubok, N. N. Zinov'ev, L. L. Samoilov, I. D. Sapozhnikov, A. V. Trukhin, and M. L. Fel'shtyn, JETP Lett. 93, 119 (2011).

${ }^{12}$ A. C. E. Chia and R. R. LaPierre, J. Appl. Phys. 112, 063705 (2012).

${ }^{13}$ V. I. Belinicher and S. M. Ryvkin, JETP 54, 190 (1981).

${ }^{14}$ J. R. Wait, Can. J. Phys. 33, 189 (1955).

${ }^{15}$ H. Chew, D. D. Cooke, and M. Kerker, Appl. Opt. 19, 44 (1980).

${ }^{16}$ A. W. Snyder, Optical Waveguide Theory (Springer, Berlin, 1983).

${ }^{17}$ S. Hu, C.-Y. Chi, K. T. Fountaine, M. Yao, H. A. Atwater, P. Daniel Dapkus, N. S. Lewis, and C. Zhou, Energy Environ. Sci. 6, 1879 (2013).

${ }^{18}$ C. F. Bohren and D. R. Huffman, Absorption and Scattering of Light by Small Particles (Wiley-VCH, 1998).

${ }^{19}$ V. N. Trukhin, A. V. Andrianov, and N. N. Zinov'ev, Phys. Rev. B 78, 155325 (2008).

${ }^{20}$ F. J. Bezares, J. P. Long, O. J. Glembocki, J. Guo, R. W. Rendell, R. Kasica, L. Shirey, J. C. Owrutsky, and J. D. Caldwel, Opt. Express 21, 27587 (2013).

${ }^{21}$ C. Lin and M. L. Povinelli, Opt. Express 17, 19371 (2009). 